\title{
On the Thermodynamic Formalism for the Gauss Map
}

\section{Dieter H. Mayer}

Max-Planck-Institut für Mathematik, Gottfried-Claren-Straße 26, D-5300 Bonn 3, Federal Republic of Germany

Abstract. We study the generalized transfer operator $\mathscr{L}_{\beta} f(z)=\sum_{n=1}^{\infty}\left(\frac{1}{z+n}\right)^{2 \beta} \times$ $f(1 /(z+n))$ of the Gauss map $T x=(1 / x) \bmod 1$ on the unit interval. This operator, which for $\beta=1$ is the familiar Perron-Frobenius operator of $T$, can be defined for $\operatorname{Re} \beta>\frac{1}{2}$ as a nuclear operator either on the Banach space $A_{\infty}(D)$ of holomorphic functions over a certain disc $D$ or on the Hilbert space $\mathscr{H}_{\mathrm{Re} \beta}^{(2)}\left(H_{-1 / 2}\right)$ of functions belonging to some Hardy class of functions over the half plane $H_{-1 / 2}$. The spectra of $\mathscr{L}_{\beta}$ on the two spaces are identical. On the space $\mathscr{H}_{\operatorname{Re} \beta}^{(2)}\left(H_{-1 / 2}\right) \mathscr{L}_{\beta}$ is isomorphic to an integral operator $\mathscr{K}_{\beta}$ with kernel the Bessel function $\mathfrak{F}_{2 \beta-1}(2 \sqrt{s t})$ and hence to some generalized Hankel transform. This shows that $\mathscr{L}_{\beta}$ has real spectrum for real $\beta>\frac{1}{2}$. On the space $A_{\infty}(D)$ the operator $\mathscr{L}_{\beta}$ can be analytically continued to the entire $\beta$-plane with simple poles at $\beta=\beta_{k}=(1-k) / 2, k=0,1,2, \ldots$ and residue the rank 1 operator $\mathscr{N}^{(k)} f=\frac{1}{2}(1 / k !) f^{(k)}(0)$. From this similar analyticity properties for the Fredholm determinant $\operatorname{det}\left(1-\mathscr{L}_{\beta}\right)$ of $\mathscr{L}_{\beta}$ and hence also for Ruelle's zeta function follow. Another application is to the function $\zeta_{M}(\beta)=\sum_{n=1}^{\infty}[n]^{\beta}$, where $[n]$ denotes the irrational $[n]=\left(n+\left(n^{2}+4\right)^{1 / 2}\right) / 2$. $\zeta_{M}(\beta)$ extends to a meromorphic function in the $\beta$-plane with the only poles at $\beta= \pm 1$ both with residue 1 .

\section{Generalized Transfer Operators for the Gauss Map}

If $I=[0,1]$ denotes the unit interval in $\mathbb{R}$ the Gauss (or continued fraction-)map $T:[0,1] \rightarrow[0,1]$ is defined as

$$
T x=\left\{\begin{array}{ll}
1 / x \bmod 1 & x \neq 0 \\
0 & x=0
\end{array} .\right.
$$

From ergodic theory for general hyperbolic systems $T: M \rightarrow M$ it is known [Bo], [Ru1] that systems like the Gauss map allow for a description in terms of symbolic dynamics $\pi: F^{\mathbb{Z}_{+}} \rightarrow M$ with an alphabet $F$ and a transition matrix $\mathbb{A}=\left(\mathbb{A}_{\sigma, \sigma^{\prime}}\right)$, $\sigma, \sigma^{\prime} \in F$, defined through a Markov partition $\mathscr{A}=\left(\mathcal{O}_{\sigma}\right)_{\sigma \in F}$. This way $T$ gets 
semi-conjugated to the shift map $\tau: F^{\mathbf{Z}_{+}} \rightarrow F^{\mathbf{Z}_{+}},(\tau \xi)_{i}=\xi_{i+1}$ for $\xi=\left(\xi_{i}\right)_{i \in \mathbf{Z}_{+}}$, on the one sided subshift of finite type with the above data. For the Gauss map $F$ turns out to be $\mathbb{N}$ and hence the symbolic dynamics takes place on a one sided subshift of infinite type. The physical system corresponding to such a subshift is a spin system on the lattice $\mathbb{Z}_{+}$whose spins take values in $\mathbb{N}$. Of special interest in the ergodic theory of hyperbolic systems are the equilibrium states [Bo] which are $T$-invariant probability measures defined through the Gibbs-ensembles of the above mentioned lattice spin systems. These Gibbs-ensembles are determined by some interaction energy characterizing the spin system [Ru1]. A rather special role from the physical point of view in this approach plays the interaction determined by the function $\varphi_{1}(x)=-\log \left|\operatorname{det} D_{u} T(x)\right|$, where $D_{u} T$ denotes the derivative of $T$ along the unstable directions of the hyperbolic system $T$ [Ru1], [Bo]. For 1-dimensional systems this is the function $\varphi_{1}(x)=-\log \left|T^{\prime}(x)\right|$, which for the Gauss map reads $\varphi_{1}(x)=\log x^{2}$. Through the symbolic dynamics $\pi: F^{\mathbf{z}_{+}} \rightarrow I$ this defines a function $A(\xi)=\varphi_{1} \circ \pi(\xi)$ in $F^{\mathbf{Z}_{+}}$. As shown in [Ma3], the map $\pi: \mathbb{N}^{\mathbf{Z}_{+}} \rightarrow I$ for the Markov partition $\mathscr{A}=\left(I_{n}\right)_{n \in \mathbf{N}}, I_{n}=[(1 / n+1),(1 / n)]$, of the Gauss map $T: I \rightarrow I$ is given by

$$
\pi(\xi)=\left[\xi_{0}, \xi_{1}, \ldots\right], \quad \text { if } \quad \xi=\left(\xi_{i}\right)_{i \in \mathbf{z}_{+}},
$$

where $\left[n_{1}, n_{2}, \ldots,\right]$ denotes the number $x \in I$ whose continued fraction expansion has entries $n_{i} \in \mathbb{N}, i \in \mathbb{Z}_{+}$. Strictly speaking, the map $\pi$ is not surjective since $\pi(\xi)$ is irrational for $\xi \in \mathbb{N}^{\mathbf{Z}}+$. This means that we are treating this way the Gauss map $T$ restricted to the invariant subset $I_{\text {inv }}=\{x \in I: x$ irrational $\}$. As long however as we are interested only in measures which are absolutely continuous with respect to Lebesgue measure the properties of $T: I_{\mathrm{inv}} \rightarrow I_{\mathrm{inv}}$ and $T: I \rightarrow I$ are the same since $I / I_{\text {inv }}$ is countable and hence has Lebesque measure zero.

A powerful method within the so-called thermodynamic formalism as developed in $[\mathrm{Ru} 1]$ is the transfer operator method. It is a straightforward generalization of the transfer matrix technique for lattice spin systems with finite range interactions [Ma2]: if $C\left(F^{\mathbf{z}_{+}}\right)$denotes the space of continuous observables of the spin system on $\mathbb{Z}_{+}$and if $A \in C\left(F^{\mathbf{z}_{+}}\right)$is any such observable, the transfer operator $\mathscr{L}: C\left(F^{\mathbf{z}_{+}}\right) \rightarrow C\left(F^{\mathbf{z}_{+}}\right)$is the following linear bounded operator:

$$
\mathscr{L} f(\xi)=\sum_{\sigma \in \boldsymbol{F}} \mathbb{A}_{\sigma, \xi_{0}} \exp A(\sigma, \xi) f(\sigma, \xi)
$$

where $(\sigma, \xi)$ denotes the configuration $\xi^{\prime}=\left(\xi_{i}^{\prime}\right)$ with $\xi_{0}^{\prime}=\sigma$ and $\xi_{i}^{\prime}=\xi_{i-1}$. If this $\xi^{\prime}$ is allowed then $\mathbb{A}_{\sigma, \xi_{0}}=1$ otherwise $\mathbb{A}_{\sigma, \xi_{0}}=0$. In the special case of a locally expanding Markov map $T: M \rightarrow M$ and the function $A=\varphi_{1}{ }^{\circ} \pi$ with $\varphi_{1}=-\log \left|\operatorname{det} T^{\prime}(x)\right|$ the operator $\mathscr{L}$ obviously induces an operator $\mathscr{L}$ on the space of observables $\tilde{f}$ of the system $T: M \rightarrow M$ which has the form

$$
\tilde{\mathscr{L}} \tilde{f}(x)=\sum_{\sigma \in F}\left|\operatorname{det} \psi_{\sigma}^{\prime}(x)\right| \tilde{f} \circ \psi_{\sigma}(x) \chi_{T o_{\sigma}}(x),
$$

and hence coincides with the so-called Perron Frobenius operator [LaM] of $T$. For the Gauss map the operator has the form [Ma1],

$$
\tilde{\mathscr{L}} \tilde{f}(x)=\sum_{n=1}^{\infty}\left(\frac{1}{x+n}\right)^{2} \tilde{f}\left(\frac{1}{x+n}\right)
$$


and its spectral properties in the Banach space $A_{\infty}(D)$ of functions holomorphic and continuous over the disc $D=\left\{z:|z-1|<\frac{3}{2}\right\}$ have been studied in [Ma1], [MaR1], [MaR2]. The main application of operators of the form (3) originally was to construct invariant measures for the system $T: M \rightarrow M$, a special case being the Sinai-Bowen-Ruelle $[\mathrm{BoR}]$ measure corresponding to the choice $A(\xi)=\varphi_{1} \circ \pi(\xi)$ with $\varphi_{1}$ as before [Ru2]. For general $A$ one gets by this construction Keane's $g$-measures $[\mathrm{K}]$. From equilibrium statistical mechanics of lattice spin systems one knows that the Gibbs states corresponding to the interaction $\beta A(\xi)$, considered now as a function of the parameter $\beta$, which corresponds in fact up to Boltzmann's constant to inverse temperature, describe the physical properties of such a system when coupled to an exterior heat bath of fixed temperature. From this one could expect that also for the dynamical system $T: M \rightarrow M$ the function $\varphi_{\beta}(x)=-\beta \log \mid \operatorname{det}\left(D_{u} T(x) \mid\right.$ respectively the corresponding observable for the spin system $A_{\beta}(\xi)=\varphi_{\beta}{ }^{\circ} \pi(\xi)$ plays a crucial role in the description of the properties of the system $T: M \rightarrow M$. The transfer operator then has the form

$$
\mathscr{L}_{\beta} f(\xi)=\sum_{\sigma \in \boldsymbol{F}} \mathbb{A}_{\sigma, \xi_{0}} \exp A_{\beta}(\sigma, \xi) f(\sigma, \xi) .
$$

Specialising to the Gauss map and going immediately over to the induced operator on $C(I)$ we get

$$
\mathscr{L}_{\beta} f(x)=\sum_{n=1}^{\infty}\left(\frac{1}{x+n}\right)^{2 \beta} f\left(\frac{1}{x+n}\right),
$$

where for reasons of simplicity we have omitted the tildes in (4). To our knowledge the first time where in ergodic theory an operator analogous to $\mathscr{L}_{\beta}$ has been used to characterize invariants of a dynamical system was in $[\mathrm{PaT}]$ for Markov chains. It was shown that the highest eigenvalue $\lambda_{1}(\beta)$ of a certain generalized transition matrix has a convergent power series around $\beta=1$ whose coefficients define invariants of the Markov chain. Instead of the eigenvalue $\lambda_{1}(\beta)$ it is more convenient to study the quantity $P(\beta)=\log \lambda_{1}(\beta)$. For real $\beta$ the eigenvalue $\lambda_{1}(\beta)$ is positive and $P(\beta)$ hence well defined. It can be analytically extended around the real axis, at least for systems where $F$ can be chosen finite: one applies simply the Ruelle-Perron-Frobenius (RPF) Theorem [W1] to $\mathscr{L}_{\beta}$ which shows that for real $\beta$ the leading eigenvalue $\lambda_{1}(\beta)$ is positive and simple. For these $\beta$-values $P(\beta)$ is nothing else than the pressure of the observable $\beta \varphi$ for the system $T: M \rightarrow M$, and hence a convex function in $\beta$ [Ru1]. $P(\beta)$ can be defined independently of the operator $\mathscr{L}_{\beta}$ in analogy to equilibrium statistical mechanics through the systems partition functions $Z_{n}(\beta \varphi)$ [Ru1]:

$$
P(\beta)=\lim _{n \rightarrow \infty} \frac{1}{n} \log Z_{n}(\beta \varphi),
$$

where

$$
Z_{n}(\beta \varphi)=\sum_{x \in \mathrm{Fix}} \exp \beta \sum_{k=0}^{n-1} \varphi\left(T^{k} x\right)
$$


and Fix $T^{n}=\left\{x \in M: T^{n} x=x\right\}$, respectively the variational principle [W2],

$$
P(\beta)=\sup _{\mu}\left\{h_{\mu}(T)+\beta \int_{M} \varphi(x) d \mu(x)\right\},
$$

where the supremum is taken over all $T$ invariant probability measures $\mu$, and $h_{\mu}(T)$ denotes the Kolmogorov-Sinai entropy of $T$ with respect to $\mu$. The measures maximizing the right-hand side in (9) are just the equilibrium measures $\mu$ for the observable $\beta \varphi$. For $\varphi=\varphi_{1}$ we recover thereby the Sinai-Bowen-Ruelle measure for the dynamical system $T: M \rightarrow M[\mathrm{BoR}][\mathrm{Ru} 2]$.

The results of Parry and Tuncel in $[\mathrm{PaT}]$ have been generalized recently by Rand et al. [Ra], $[\mathrm{RaB}]$ who showed how practically all quantities introduced over the last years to characterize chaotic behaviour of hyperbolic systems like entropies, dimensions, singularity spectra etc. can be derived from the function $P(\beta)$. In complete analogy to equilibrium statistical mechanics where the quantity $P(\beta)$ is up to sign and a factor of $\beta$ just the free energy of the spin system attached to the map $T$ through its symbolic dynamics, the analytic behaviour of $P(\beta)$ as a function of $\beta$ is used to define phase transitions for the dynamical system $T: M \rightarrow M$. It is clear from the RPF-Theorem that for hyperbolic systems with finite $F$ no such phase transition can occur for real $\beta$. The situation changes completely if $F$ is countable infinite, as we will show in the special case of the Gauss map. Similar results are expected to hold for all hyperbolic systems with a countable infinite Markov partition. Candidates are the higher dimensional versions of the continued fraction transformation as for instance the Jacobi-Perron algorithm. Their transfer operators for $\beta=1$ have been discussed in [Ma5].

In this paper we restrict the discussion to the ordinary Gauss map in dimension 1. It would be interesting to see how far our results for this case can be extended to higher dimensions. By applying the same arguments as in [Ma1] respectively [MaR1], [MaR2] one shows for general $\beta \in \mathbb{C}$ with $\operatorname{Re} \beta>\frac{1}{2}$ that for a discussion of the pressure $P(\beta)$ of the Gauss map one can restrict the generalized transfer operator $\mathscr{L}_{\beta}$ in (6) to the Banach space $A_{\infty}(D)$ of holomorphic functions over the domain $D$ defined as for the operator $\mathscr{L}_{1}=\mathscr{L}$ in (4). As for $\beta=1$ one has

Proposition 1. The operator $\mathscr{L}_{\beta}: A_{\infty}(D) \rightarrow A_{\infty}(D)$ with

$$
\mathscr{L}_{\beta} f(z)=\sum_{n=1}^{\infty}\left(\frac{1}{z+n}\right)^{2 \beta} f\left(\frac{1}{z+n}\right)
$$

is nuclear of order zero for $\beta \in \mathbb{C}$ with $\operatorname{Re} \beta>\frac{1}{2}$. For real $\beta>\frac{1}{2} \mathscr{L}_{\beta}$ has a leading positive eigenvalue $\lambda_{1}(\beta)$ which is simple such that $P(\beta)=\log \lambda_{1}(\beta)$ is the pressure of the observable $\varphi_{\beta}$ for the Gauss map. For any $m \in \mathbb{N}$ the following trace formula holds:

$$
\operatorname{trace} \mathscr{L}_{\beta}^{m}=\sum_{x \in \mathrm{Fix}} \frac{\prod_{k=0}^{m-1}\left(T^{k} x\right)^{2 \beta}}{1-(-1)^{m} \prod_{k=0}^{m-1}\left(T^{k} x\right)^{2}} .
$$


As a consequence we hence get for the partition functions

$$
Z_{n}(\beta)=\sum_{x \in \text { Fix } T^{n}} \exp \sum_{k=0}^{n-1} \varphi_{\beta}\left(T^{k} x\right):
$$

Corollary 1. $Z_{n}(\beta)=$ trace $\mathscr{L}_{\beta}^{(0) n}$ - trace $\mathscr{L}_{\beta}^{(1) n}$, where the generalized transfer operators $\mathscr{L}_{\beta}^{(s)}, s=0,1$, are defined as

$$
\mathscr{L}_{\beta}^{(s)} f(z)=(-1)^{s} \sum_{n=1}^{\infty}\left(\frac{1}{z+n}\right)^{2(\beta+s)} f\left(\frac{1}{z+n}\right) .
$$

Obviously $\mathscr{L}_{\beta}^{(0)}=\mathscr{L}_{\beta}$ and $\mathscr{L}_{\beta}^{(1)}=-\mathscr{L}_{\beta+1}$ with $\mathscr{L}_{\beta}$ as in Proposition 1 . Since the map $\beta \rightarrow \mathscr{L}_{\beta}^{(s)}$ is holomorphic in $\beta$ for $\operatorname{Re}(\beta+s)>\frac{1}{2}$ we find that the function

$$
\zeta(\beta)=\exp \sum_{n=1}^{\infty} \frac{Z_{n}(\beta)}{n}
$$

which by a standard argument can be written as

$$
\zeta(\beta)=\frac{\operatorname{det}\left(1-\mathscr{L}_{\beta}^{(1)}\right)}{\operatorname{det}\left(1-\mathscr{L}_{\beta}^{(0)}\right)}
$$

is a meromorphic function in the domain $\operatorname{Re} \beta>\frac{1}{2}$. Thereby one makes use of the fact that the Fredholm determinant $\operatorname{det}\left(1-\mathscr{L}_{\beta}^{(s)}\right)$ is a holomorphic function of $\beta$ in the domain where $\mathscr{L}_{\beta}^{(s)}$ depends holomorphically on $\beta$ [G]. Obviously, the function $\zeta(\beta)$ has poles in the domain $\operatorname{Re} \beta>\frac{1}{2}$ among those $\beta$ values where $\mathscr{L}_{\beta}^{(0)}$ has $\lambda=1$ among its eigenvalues. This is certainly the case for $\beta=1$ where $\lambda=1$ is the leading eigenvalue of $\mathscr{L}_{1}^{(0)}$ and hence is simple. Since in this case $\mathscr{L}_{\beta}^{(1)}$ is just the operator $-\mathscr{L}_{2}^{(0)}$, whose leading eigenvalue is strictly smaller than 1 in absolute value, the Fredholm determinant $\operatorname{det}\left(1-\mathscr{L}_{1}^{(1)}\right) \neq 0$ and hence $\beta=1$ is a simple pole of $\zeta(\beta)$.

Problem. What is the residue of $\zeta(\beta)$ at $\beta=1$ ?

It is one of the aims of this paper to show that the function $\zeta(\beta)$ in (13) can be extended to the entire $\beta$-plane and defines there a meromorphic function. Before doing this we want to relate the generalized transfer operators $\mathscr{L}_{\beta}^{(s)}$ in (11) to some integral operator with a simple kernel acting in some Hilbert space of square summable functions. The resulting integral transform turns out to be a generalized Hankel transformation. This extends an analogous result for the case $\beta=1$ discussed in [MaR1], [MaR2]. Since the arguments for general $\beta, \operatorname{Re} \beta>\frac{1}{2}$ are slightly more complicated than for $\beta=1$ we present them in more detail in the next section.

\section{The Operators $\mathscr{L}_{\beta}^{(s)}$ in Generalized Hardy Spaces}

Since $\mathscr{L}_{\beta}^{(1)}=-\mathscr{L}_{\beta+1}^{(0)}$ it is enough to discuss the operator $\mathscr{L}_{\beta}^{(0)}$ which for simplicity we denote again by $\mathscr{L}_{\beta}$. If $f \in A_{\infty}(D)$ is an eigenfunction of $\mathscr{L}(\beta)$ then one deduces recursively from the eigenequation

$$
\lambda f(z)=\sum_{n=1}^{\infty}\left(\frac{1}{z+n}\right)^{2 \beta} f\left(\frac{1}{z+n}\right)=\mathscr{L}_{\beta} f(z)
$$


that $f$ can be extended to a function holomorphic in the entire complex $z$ plane cut along the line $(-\infty,-1]$. This follows from the contraction properties of the maps $\psi_{n}(z)=1 /(z+n)$. If we therefore denote by $H_{\delta}$ the half plane

$$
H_{\delta}=\{z \in \mathbb{C}: \operatorname{Re} z>\delta\},
$$

then any eigenfunction $f$ of $\mathscr{L}_{\beta}$ in $A_{\infty}(D)$ is holomorphic in every half plane $H_{-1+\varepsilon}$ for any $\varepsilon>0$. It is also evident from Eq. (14) that any such eigenfunction vanishes for $|z| \rightarrow \infty$ in these half planes, and hence is also bounded there. From this one can expect that $\mathscr{L}_{\beta}$ can be defined on some Hardy space of functions holomorphic in a half plane. We denote by $\mathscr{H}^{(2)}\left(H_{\delta}\right)$ the ordinary Hardy space of functions holomorphic over the half plane $H_{\delta}[\mathrm{D}]$ :

$$
\begin{aligned}
\mathscr{H}^{(2)}\left(H_{\delta}\right)= & \left\{f: f \text { holomorphic in } H_{\delta}, f\right. \text { bounded in } \\
& \left.H_{\delta+\varepsilon} \text { for all } \varepsilon>0 \text { and } \int_{-\infty}^{+\infty}|f(\delta+i y)|^{2} d y<\infty\right\} .
\end{aligned}
$$

This space is known to be a Hilbert space with scalar product

$$
\left(f_{1}, f_{2}\right)=\frac{1}{2 \pi} \int_{-\infty}^{+\infty} f_{1}^{*}(\delta+i y) f_{2}(\delta+i y) d y
$$

Furthermore the Paley-Wiener Theorem holds, giving a simple characterization of the elements of this space [D]:

Theorem 1. A function $f$ belongs to the space $\mathscr{H}^{(2)}\left(H_{\delta}\right)$ if and only if there exists a function $\varphi \in \mathscr{L}_{2}\left(d s, \mathbb{R}_{+}\right)$such that

$$
f(z)=\int_{0}^{\infty} d s e^{-s z} e^{\delta s} \varphi(s) .
$$

The function $\varphi$ is unique (in the $\mathscr{L}_{2}$ sense) and

$$
\|f\|_{\mathscr{H}^{(2)}\left(H_{\delta}\right)}^{2}=\frac{1}{2 \pi} \int_{-\infty}^{+\infty}|f(\delta+i y)|^{2} d y=\int_{0}^{\infty} d s|\varphi(s)|^{2}=\|\varphi\|_{\mathscr{L}_{2}}^{2} .
$$

From what we have found earlier for the eigenfunctions of $\mathscr{L}_{\beta}$ one could expect that the Hardy space $\mathscr{H}^{(2)}\left(H_{\delta}\right)$ for appropriately chosen $\delta>-1$ would be a good space for $\mathscr{L}_{\beta}$ to be defined on. The discussion of the case $\beta=1$ in [MaR1] shows however that a slightly modified class of functions is better for simplifying the operator $\mathscr{L}_{\beta}$. Generalizing the procedure in [MaR1] we define for arbitrary real $\alpha \geqq 1$ the space $\mathscr{H}_{\alpha}^{(2)}\left(H_{\delta}\right)$ as follows:

$$
\begin{aligned}
\mathscr{H}_{\alpha}^{(2)}\left(H_{\delta}\right)= & \left\{f: f \text { holom. in } H_{\delta}, f \in \mathscr{H}^{(2)}\left(H_{\delta+\varepsilon}\right) \text { for any } \varepsilon>0\right. \text { and } \\
& \left.\int_{0}^{\infty} x^{2 \alpha-2} d x \int_{-\infty}^{+\infty} d y\left(|f(x+\delta+i y)|^{2}-\left|f\left(x+\delta+\frac{1}{2}+i y\right)\right|^{2}\right)<\infty\right\} .
\end{aligned}
$$


We want to show that $\mathscr{H}_{\alpha}^{(2)}\left(H_{\delta}\right)$ is a Hilbert space. Since any $f \in \mathscr{H}_{\alpha}^{(2)}\left(H_{\delta}\right)$ has the property that $\int_{-\infty}^{+\infty} d y|f(\delta+x+i y)|^{2}<\infty$ for $x>0$ we find that for any $\varepsilon>0$ and
$N<\infty$,

$$
\begin{aligned}
& \int_{\varepsilon}^{N} x^{2 \alpha-2} d x \int_{-\infty}^{+\infty} d y\left(|f(x+\delta+i y)|^{2}-\left|f\left(x+\delta+\frac{1}{2}+i y\right)\right|^{2}\right) \\
& =\int_{\varepsilon}^{N} x^{2 \alpha-2} d x \int_{-\infty}^{+\infty} d y|f(x+\delta+i y)|^{2}-\int_{\varepsilon}^{N} x^{2 \alpha-2} d x \int_{-\infty}^{+\infty} d y\left|f\left(x+\delta+\frac{1}{2}+i y\right)\right|^{2} .
\end{aligned}
$$

Changing in the first term of the right-hand side the variable $x$ to $x=x^{\prime}+\frac{1}{2}$ gives

$$
\begin{aligned}
\text { left-hand side }= & \int_{-(1 / 2)+\varepsilon}^{-(1 / 2)+N}\left(x^{\prime}+\frac{1}{2}\right)^{2 \alpha-2} d x^{\prime} \int_{-\infty}^{+\infty} d y\left|f\left(x^{\prime}+\frac{1}{2}+\delta+i y\right)\right|^{2} \\
& -\int_{\varepsilon}^{N} x^{2 \alpha-2} d x \int_{-\infty}^{+\infty} d y\left|f\left(x+\delta+\frac{1}{2}+i y\right)\right|^{2} .
\end{aligned}
$$

Introducing next the function $\varphi_{\alpha, N}^{(\varepsilon)}(x)$ with

$$
\varphi_{\alpha, N}^{(\varepsilon)}(x)=\left\{\begin{array}{ll}
0 & x<-\frac{1}{2}+\varepsilon, \quad x>N \\
\left(x+\frac{1}{2}\right)^{2 \alpha-2} & -\frac{1}{2}+\varepsilon \leqq x \leqq \varepsilon \\
\left(x+\frac{1}{2}\right)^{2 \alpha-2}-x^{2 \alpha-2} & \varepsilon \leqq x \leqq-\frac{1}{2}+N \\
-x^{2 \alpha-2} & -\frac{1}{2}+N \leqq x \leqq N
\end{array},\right.
$$

we have shown for any $\varepsilon>0, N<\infty$

$$
\begin{gathered}
\int_{\varepsilon}^{N} x^{2 \alpha-2} d x \int_{-\infty}^{+\infty} d y\left(|f(x+\delta+i y)|^{2}-\left|f\left(x+\delta+\frac{1}{2}+i y\right)\right|^{2}\right) \\
=\int_{-\infty}^{+\infty} \int_{-\infty}^{+\infty} \varphi_{\alpha, N}^{(\varepsilon)}(x) d x d y\left|f\left(x+\frac{1}{2}+\delta+i y\right)\right|^{2} .
\end{gathered}
$$

For $f \in \mathscr{H}_{\alpha}^{(2)}\left(H_{\delta}\right)$ the limit $\varepsilon \rightarrow 0, N \rightarrow \infty$ on the left-hand side exists, and hence also the limit on the right-hand side exists and the two coincide. Since $\varphi_{\alpha, N}^{(\varepsilon)}$ converges for $\varepsilon \rightarrow 0, N \rightarrow \infty$ to the positive density $\varphi_{\alpha}(x)$, where

$$
\varphi_{\alpha}(x)= \begin{cases}0 & x<-\frac{1}{2} \\ \left(x+\frac{1}{2}\right)^{2 \alpha-2} & -\frac{1}{2} \leqq x \leqq 0, \\ \left(x+\frac{1}{2}\right)^{2 \alpha-2}-x^{2 \alpha-2} & 0 \leqq x\end{cases}
$$

we have shown that for $f \in \mathscr{H}_{\alpha}^{(2)}\left(H_{\delta}\right)$,

$$
\begin{gathered}
\int_{0}^{\infty} x^{2 \alpha-2} d x \int_{-\infty}^{+\infty} d y\left(|f(x+\delta+i y)|^{2}-\left|f\left(x+\delta+\frac{1}{2}+i y\right)\right|^{2}\right) \\
=\int_{-\infty}^{+\infty} \varphi_{\alpha}(x) d x \int_{-\infty}^{+\infty} d y\left|f\left(x+\delta+\frac{1}{2}+i y\right)\right|^{2} .
\end{gathered}
$$

This shows that $\mathscr{H}_{\alpha}^{(2)}\left(H_{\delta}\right)$ is indeed a Hilbert space.

The Paley-Wiener Theorem for this space takes the following form:

Theorem 2. A function $f$ belongs to the space $\mathscr{H}_{\alpha}^{(2)}\left(H_{\delta}\right)$ if and only if there exists a 
function $\varphi \in \mathscr{L}_{2}\left(d s, \mathbb{R}_{+}\right)$such that

$$
f(z)=\int_{0}^{\infty} d s e^{-s z} e^{s \delta} s^{\alpha-1 / 2}\left(1-e^{-s}\right)^{-1 / 2} \varphi(s)
$$

The function $\varphi$ is unique in the $\mathscr{L}_{2}$-sense and

$$
\begin{aligned}
\|f\|_{\mathscr{H}_{\alpha}^{(2)}\left(H_{\delta}\right)}^{2} & =\frac{1}{2 \pi} \int_{0}^{\infty} x^{2 \alpha-2} d x \int_{-\infty}^{+\infty} d y\left(|f(x+\delta+i y)|^{2}-\left|f\left(x+\delta+\frac{1}{2}+i y\right)\right|^{2}\right) \\
& =\frac{\Gamma(2 \alpha-1)}{2^{2 \alpha-1}} \int_{0}^{\infty} d s|\varphi(s)|^{2} .
\end{aligned}
$$

Proof. The "if" - part of Theorem 2 is easy: assume $f$ can be written as in (22). Since the function $g_{\varepsilon}(s)=e^{-\varepsilon s} s^{\alpha-1 / 2}(1-e)^{-1 / 2}$ is bounded on the real line $0 \leqq s<\infty$ for any $\varepsilon>0$ the function $g_{\varepsilon}(s) \varphi(s)$ belongs to $\mathscr{L}_{2}\left(d s, \mathbb{R}_{+}\right)$. The Paley-Wiener Theorem then implies that the function

$$
f(z)=\int_{0}^{\infty} d s e^{-s z} e^{s(\delta+\varepsilon)} g_{\varepsilon}(s) \varphi(s)
$$

belongs to $\mathscr{H}^{(2)}\left(H_{\delta+\varepsilon}\right)$ for any $\varepsilon>0$. Furthermore any such $f$ obviously is holomorphic in $H_{\delta}$. Applying next for $x>\delta$ Plancherel's Theorem to the function $f_{x}(y)=f(x+i y)$ we find

$$
\frac{1}{2 \pi} \int_{-\infty}^{+\infty}|f(x+i y)|^{2} d y=\int_{0}^{\infty} d s e^{-2 s(x-\delta)} s^{2 \alpha-1}\left(1-e^{-s}\right)^{-1}|\varphi(s)|^{2},
$$

respectively for $x>0$,

$$
\frac{1}{2 \pi} \int_{-\infty}^{+\infty}|f(x+\delta+i y)|^{2} d y=\int_{0}^{\infty} d s e^{-2 s x} s^{2 \alpha-1}\left(1-e^{-s}\right)^{-1}|\varphi(s)|^{2} .
$$

From this we conclude that for $x>0$,

$$
\frac{1}{2 \pi} \int_{-\infty}^{+\infty}\left(|f(x+\delta+i y)|^{2}-\left|f\left(x+\delta+\frac{1}{2}+i y\right)\right|^{2}\right) d y=\int_{0}^{\infty} d s s^{2 \alpha-1} e^{-2 s x}|\varphi(s)|^{2},
$$

and hence

$$
\begin{aligned}
& \frac{1}{2 \pi} \int_{0}^{\infty} x^{2 \alpha-2} d x \int_{-\infty}^{+\infty}\left(|f(x+\delta+i y)|^{2}-\left|f\left(x+\delta+\frac{1}{2}+i y\right)\right|^{2}\right) d y \\
& \quad=\int_{0}^{\infty} d s s^{2 \alpha-1}|\varphi(s)|^{2} \int_{0}^{\infty} x^{2 \alpha-2} e^{-2 s x} d x=\frac{\Gamma(2 \alpha-1)}{2^{2 \alpha-1}} \int_{0}^{\infty} d s|\varphi(s)|^{2} .
\end{aligned}
$$

To prove the "only if" part of Theorem 2 we proceed as follows: for $\eta>0$ we define for any $f \in \mathscr{H}_{\alpha}^{(2)}\left(H_{\delta}\right)$ the function

Then we have

$$
f_{\eta}(z)=f(\eta+z)
$$

Lemma 1. For any $f \in \mathscr{H}_{\alpha}^{(2)}\left(H_{\delta}\right)$ and $\eta>0$ the function $f_{\eta}$ is in $\mathscr{H}_{\alpha}^{(2)}\left(H_{\delta}\right) \cap \mathscr{H}^{(2)}\left(H_{\delta}\right)$ and $\lim _{\eta \rightarrow 0} f_{\eta}=f$, where convergence is in the space $\mathscr{H}_{\alpha}^{(2)}\left(H_{\delta}\right)$. 
Proof of Lemma 1. By definition any $f \in \mathscr{H}_{\alpha}^{(2)}\left(H_{\delta}\right)$ belongs to the space $\mathscr{H}^{(2)}\left(H_{\delta+\eta}\right)$ for any $\eta>0$. By Paley-Wiener this means $f(z)=\int_{0}^{\infty} e^{-s z} e^{(\delta+\eta) s} \varphi_{\eta}(s) d s$ with $\varphi_{\eta} \in \mathscr{L}_{2}\left(d s, \mathbb{R}_{+}\right)$. Hence $f_{\eta}(z)=\int_{0}^{\infty} e^{-s z} e^{\delta s} \varphi_{\eta}(s) d s$ belongs to $\mathscr{H}^{(2)}\left(H_{\delta}\right)$ and trivially also to $\mathscr{H}^{(2)}\left(H_{\delta+\varepsilon}\right)$ for any $\varepsilon>0$. To prove that $f_{\eta} \in \mathscr{H}_{\alpha}^{(2)}\left(H_{\delta}\right)$, we have to show that $\left\|f_{\eta}\right\|_{\mathscr{H}_{\alpha}^{(2)}\left(H_{\delta}\right)}^{2}<\infty$. But

$$
\begin{aligned}
& \int_{0}^{\infty} x^{2 \alpha-2} d x \int_{-\infty}^{+\infty} d y\left(|f(x+\delta+\eta+i y)|^{2}-\left|f\left(x+\delta+\eta+\frac{1}{2}+i y\right)\right|^{2}\right) \\
& =\int_{\eta}^{\infty}\left(x^{\prime}-\eta\right)^{2 \alpha-2} d x^{\prime} \int_{-\infty}^{+\infty} d y\left(\left|f\left(x^{\prime}+\delta+i y\right)\right|^{2}-\left|f\left(x^{\prime}+\delta+\frac{1}{2}+i y\right)\right|^{2}\right) .
\end{aligned}
$$

Since $\alpha \geqq 1$ and $\int_{-\infty}^{+\infty} d y\left|f\left(x^{\prime}+\delta+i y\right)\right|^{2} \geqq \int_{-\infty}^{+\infty}\left|f\left(x^{\prime}+\delta+\frac{1}{2}+i y\right)\right|^{2}$ for $x^{\prime}>0[\mathrm{D}]$ we find for any $\eta \geqq 0$ :

$$
\left\|f_{\eta}\right\|_{\mathscr{H}_{\alpha}^{(2)}\left(H_{\delta}\right)}^{2} \leqq\|f\|_{\mathscr{H}_{\alpha}^{(2)}\left(H_{\delta}\right)}^{2}
$$

To show finally that $f_{\eta}$ converges to $f$ in the space $\mathscr{H}_{\alpha}^{(2)}\left(H_{\delta}\right)$ we argue as follows: since $f-f_{\eta} \in \mathscr{H}_{\alpha}^{(2)}\left(H_{\delta}\right)$ we know from relation (21) that

$$
\int_{-\infty}^{+\infty} \varphi_{\alpha}(x) d x \int_{-\infty}^{+\infty} d y\left|f\left(x+\delta+\frac{1}{2}+i y\right)-f\left(x+\delta+\eta+\frac{1}{2}+i y\right)\right|^{2}<\infty .
$$

Hence there exists for any $\varepsilon>0$ a compact set $K_{\varepsilon}$ such that both $f$ and $f_{\eta}$ are holomorphic in $K_{\varepsilon}$ and

$$
\int_{\mathbb{R}^{2} \backslash K_{\varepsilon}} \varphi_{\alpha}(x) d x d y\left|f\left(x+\delta+\frac{1}{2}+i y\right)-f\left(x+\delta+\eta+\frac{1}{2}+i y\right)\right|^{2}<\frac{\varepsilon}{2} .
$$

On the other hand there exists $\eta=\eta(\varepsilon)$ such that

$$
\sup _{z \in K_{\varepsilon}}\left|f\left(z+\delta+\frac{1}{2}\right)-f\left(z+\eta+\delta+\frac{1}{2}\right)\right|<\frac{\varepsilon}{2}\left(\int_{K_{\varepsilon}} \varphi_{\alpha}(x) d x d y\right)^{-1},
$$

and hence $\lim _{\eta \rightarrow 0}\left\|f-f_{\eta}\right\|_{\mathscr{H}_{\alpha}^{(2)}\left(H_{\delta}\right)}^{2}=0$ follows. This concludes the proof of Lemma 1.

To proceed then with the proof of Theorem 2 we apply again the Paley-Wiener Theorem, now to the function $f_{\eta}$ which by Lemma 1 belongs to $\mathscr{H}^{(2)}\left(H_{\delta}\right)$ for $\eta>0$. Hence there exists a function $\psi_{\eta} \in \mathscr{L}_{2}\left(d s, \mathbb{R}_{+}\right)$such that

$$
f_{\eta}(z)=\int_{0}^{\infty} d s e^{-s z} e^{\delta s} \psi_{\eta}(s)
$$

Plancherel's Theorem then gives for all $x \geqq \delta$,

$$
\frac{1}{2 \pi} \int_{-\infty}^{+\infty}\left|f_{\eta}(x+i y)\right|^{2} d y=\int_{0}^{\infty} d s e^{-2 s x} e^{2 \delta s}\left|\psi_{\eta}(s)\right|^{2} .
$$

Since $f_{\eta} \in \mathscr{H}_{\alpha}^{(2)}\left(H_{\delta}\right)$ we have

$$
\int_{0}^{\infty} x^{2 \alpha-2} d x \int_{-\infty}^{+\infty}\left(\left|f_{\eta}(x+\delta+i y)\right|^{2}-\left|f_{\eta}\left(x+\delta+\frac{1}{2}+i y\right)\right|^{2}\right) d y<\infty .
$$


Together with relation (28) this shows that

$$
\int_{0}^{\infty} x^{2 \alpha-2} d x \int_{0}^{\infty} d s e^{-2 s x}\left(1-e^{-s}\right)\left|\psi_{\eta}(s)\right|^{2} d s<\infty,
$$

and hence by Fubini

$$
\int_{0}^{\infty} d s\left(1-e^{-s}\right)\left|\psi_{\eta}(s)\right|^{2} \int_{0}^{\infty} d x x^{2 \alpha-2} e^{-2 s x}<\infty
$$

The $x$-integration can be performed to give $\left(\Gamma(2 \alpha-1) / 2^{2 \alpha-1}\right) s^{-(2 \alpha-1)}$. From (29) therefore we deduce that there must exist a function $\varphi_{\eta} \in \mathscr{L}_{2}\left(d s, \mathbb{R}_{+}\right)$such that

$$
\psi_{\eta}(s)=\varphi_{\eta}(s) s^{\alpha-1 / 2}\left(1-e^{-s}\right)^{-1 / 2} .
$$

From this we conclude that the function $f_{\eta}=f(z+\eta)$ for $\eta>0$ has the unique representation

$$
f_{\eta}(z)=\int_{0}^{\infty} d s e^{-s z} e^{\delta s} s^{\alpha-1 / 2}\left(1-e^{-s}\right)^{-1 / 2} \varphi_{\eta}(s)
$$

such that

$$
\frac{1}{2 \pi} \int_{\mathbf{R}^{2}} \varphi_{\alpha}(x) d x d y\left|f_{\eta}\left(z+\delta+\frac{1}{2}\right)\right|^{2}=\frac{\Gamma(2 \alpha-1)}{2^{2 \alpha-1}} \int_{0}^{\infty} d s\left|\varphi_{\eta}(s)\right|^{2} .
$$

Since by Lemma 1 the sequence $\left\{f_{\eta}\right\}$ is a Cauchy-sequence, it follows from (32) that also $\left\{\varphi_{\eta}\right\}$ is a Cauchy sequence in $\mathscr{L}_{2}\left(d s, \mathbb{R}_{+}\right)$. Hence there exists a unique $\varphi \in \mathscr{L}_{2}\left(d s, \mathbb{R}_{+}\right)$with $\lim _{\eta \rightarrow 0} \varphi_{\eta}=\varphi$ in the $\mathscr{L}_{2}$ sense. Define finally the function

$$
\tilde{f}(z)=\int_{0}^{\infty} d s e^{-s z} e^{\delta s} s^{\alpha-1 / 2}\left(1-e^{-s}\right)^{-1 / 2} \varphi(s)
$$

which by the first part of Theorem 1 belongs to $\mathscr{H}_{\alpha}^{(2)}\left(H_{\delta}\right)$. From relation (32) applied to the function $\tilde{f}-f_{\eta}$ we get

$$
\lim _{\eta \rightarrow 0} f_{\eta}=\tilde{f}
$$

But by Lemma 1 we also have $\lim _{\eta \rightarrow 0} f_{\eta}=f$, and hence

$$
f(z)=\tilde{f}(z)=\int_{0}^{\infty} d s e^{-s z} e^{\delta s} s^{\alpha-1 / 2}\left(1-e^{-s}\right)^{-1 / 2} \varphi(s)
$$

with an unique $\varphi \in \mathscr{L}_{2}\left(d s, \mathbb{R}_{+}\right)$. This concludes the proof of Theorem 2.

Equivalent to Theorem 2 is the following

Corollary 2. A function $f$ belongs to the space $\mathscr{H}_{\alpha}^{(2)}\left(H_{\delta}\right)$ if and only if there exists $\hat{\varphi} \in \mathscr{L}_{2}\left(d m, \mathbb{R}_{+}\right)$such that

$$
f(z)=\int^{\infty} d m(s) e^{-s z} e^{(\delta+1 / 2) s} s^{\alpha-1 / 2} \hat{\varphi}(s), \quad \text { where } \quad d m(s)=\frac{d s}{e^{s}-1} .
$$

The function $\hat{\varphi}$ is unique and

$$
\|f\|_{\mathscr{H}_{\alpha}^{(2)}\left(H_{\delta}\right)}^{2}=\frac{\Gamma(2 \alpha-1)}{2^{2 \alpha-1}} \int_{0}^{\infty} d m(s)|\hat{\varphi}(s)|^{2} .
$$


Proof. Set $\hat{\varphi}(s)=\left(e^{s}-1\right)^{1 / 2} \varphi(s)$.

In (18) we defined generalized Hardy spaces $H_{\alpha}^{(2)}\left(H_{\delta}\right)$ for real $\alpha \geqq 1$. This definition can be extended to $\alpha$ 's with $\alpha>\frac{1}{2}$ as follows: if $\alpha \geqq 1$ then any function $f$ in $\mathscr{H}_{\alpha}^{(2)}\left(H_{\delta}\right)$ obviously has the property that its derivative $f^{\prime}$ with $f^{\prime}(z)=(d / d z) f(z)$ belongs to the space $\mathscr{H}_{\alpha+1}^{(2)}\left(H_{\delta}\right)$. Hence we can define for arbitrary $\alpha$ with $\frac{1}{2}<\alpha<1$,

$$
\mathscr{H}_{\alpha}^{(2)}\left(H_{\delta}\right)=\left\{f: f \text { holom. in } H_{\delta}, \lim _{x \rightarrow \infty} f(x+i y)=0 \text { and } f^{\prime} \in \mathscr{H}_{\alpha+1}^{(2)}\left(H_{\delta}\right)\right\} .
$$

Then it is clear that Theorem 2 respectively Corollary 2 hold for general real $\alpha$ with $\alpha>\frac{1}{2}$.

If one wanted to define the spaces $\mathscr{H}_{\alpha}^{(2)}\left(H_{\delta}\right)$ also for $\alpha \leqq \frac{1}{2}$ the situation gets more complicated as one can see already from representation (22). For such $\alpha$-values the function $e^{-s(x-\delta)} s^{\alpha-1 / 2}\left(1-e^{-s}\right)^{-1 / 2}$ is for $x>\delta$, because of the singularity in $s=0$, not any more bounded, even not $\mathscr{L}_{2}$ on $[0, \infty)$. Hence such a representation can be interpreted in the whole half plane $H_{\delta}$ only in the sense of distributions. Since for our present discussion we do not need values $\alpha<\frac{1}{2}$, we do not enter this problem here. The following result shows how the above spaces $\mathscr{H}_{\alpha}^{(2)}\left(H_{\delta}\right)$ are related to the generalized transfer operators $\mathscr{L}_{\beta}$ of the Gauss map:

Theorem 3. If $\mathscr{L}_{\beta}: \mathscr{H}_{\operatorname{Re} \beta}^{(2)}\left(H_{-1 / 2}\right) \rightarrow \mathscr{H}_{\operatorname{Re} \beta}^{(2)}\left(H_{-1 / 2}\right)$ denotes the operator

$$
\mathscr{L}_{\beta} f(z)=\sum_{n=1}^{\infty}\left(\frac{1}{z+n}\right)^{2 \beta} f\left(\frac{1}{z+n}\right) \quad \text { and } \quad \mathscr{K}_{\beta}: \mathscr{L}_{2}\left(d m, \mathbb{R}_{+}\right) \rightarrow \mathscr{L}_{2}\left(d m, \mathbb{R}_{+}\right)
$$

denotes the integral operator $\mathscr{K}_{\beta} \varphi(s)=\int_{0}^{\infty} d m(t) \mathfrak{\mho}_{2 \beta-1}(2 \sqrt{s t}) \varphi(t)$ with $d m(t)=$ $d t /\left(e^{t}-1\right)$, then the two operators are isomorphic for all $\beta \in \mathbb{C}$ with $\operatorname{Re} \beta>\frac{1}{2}$. They are both of trace class.

Hence we have also

Corollary 3. The operators $\mathscr{L}_{\beta}$ and $\mathscr{K}_{\beta}$ have the same spectrum, that means all eigenvalues are identical and have the same multiplicity. For real $\beta$ they are real.

Proof of Theorem 3. For fixed $\beta=\beta_{1}+i \beta_{2}$ by Corollary 2 any element $f$ of the space $\mathscr{H}_{\beta_{1}}^{(2)}\left(H_{-1 / 2}\right)$ has an essentially unique representation as

$$
f(z)=\int_{0}^{\infty} d m(s) e^{-s z} s^{\beta-1 / 2} \hat{\varphi}(s)
$$

with $\hat{\varphi} \in \mathscr{L}_{2}\left(d m, \mathbb{R}_{+}\right)$and $d m(s)=d s /\left(e^{s}-1\right)$. There we have taken out a factor $s^{i \beta_{2}}$ from the function $\hat{\varphi}$ of that Corollary. Applying the operator $\mathscr{L}_{\beta}$ to such a $f$ we find

$$
\mathscr{L}_{\beta} f(z)=\sum_{n=1}^{\infty}\left(\frac{1}{z+n}\right)^{2 \beta} \int_{0}^{\infty} d m(s) s^{\beta-1 / 2} \hat{\varphi}(s) e^{-s /(z+n)} .
$$

Absolute convergence of both the sum and the integral allows us to interchange summation and integration in (37) to get

$$
\mathscr{L}_{\beta} f(z)=\int_{0}^{\infty} d m(s) s^{\beta-1 / 2} \hat{\varphi}(s) \sum_{n=1}^{\infty}\left(\frac{1}{z+n}\right)^{2 \beta} e^{-s /(z+n)} .
$$


The sum under the integral can be rewritten by using the Hurwitz zeta function

as follows:

$$
\zeta(s, z)=\sum_{n=0}^{\infty}\left(\frac{1}{z+n}\right)^{s}
$$

$$
\sum_{n=1}^{\infty}\left(\frac{1}{z+n}\right)^{2 \beta} e^{-s /(z+n)}=\sum_{k=0}^{\infty} \frac{(-s)^{k}}{k !} \zeta(k+2 \beta, z+1) .
$$

For $\operatorname{Re} s>1$ on the other hand the function $\zeta(s, z)$ has the integral representation [GR]

$$
\zeta(s, z)=\frac{1}{\Gamma(s)} \int_{0}^{\infty} \frac{t^{s-1} e^{-(z-1) t}}{e^{t}-1} d t,
$$

so that the right-hand side in (40) can be written as

$$
\text { right-hand side of }(40)=\sum_{k=0}^{\infty} \frac{(-s)^{k}}{k !} \frac{1}{\Gamma(k+2 \beta)} \int_{0}^{\infty} t^{k+2 \beta-1} e^{-z t} d m(t)
$$

or, after performing the $k$ summation [GR]:

$$
\sum_{k=0}^{\infty} \frac{(-s t)^{k}}{k ! \Gamma(k+2 \beta)}=\frac{\mathfrak{F}_{2 \beta-1}(2 \sqrt{t s})}{(t \cdot s)^{\beta-1 / 2}}
$$

where $\mathfrak{F}_{\beta}$ denotes the Bessel function of order $\beta$, we find

$$
\text { right-hand side of }(40)=s^{1 / 2-\beta} \int_{0}^{\infty} t^{\beta-1 / 2} e^{-z t} \mathfrak{F}_{2 \beta-1}(2 \sqrt{s t}) \text {. }
$$

Inserting this into relation (38) we therefore get

$$
\mathscr{L}_{\beta} f(z)=\int_{0}^{\infty} d m(s) \hat{\varphi}(s) \int_{0}^{\infty} d m(t) t^{\beta-1 / 2} e^{-z t} \mathfrak{\mho}_{2 \beta-1}(2 \sqrt{s t}) .
$$

Interchanging once more the order of integration we finally arrive at

$$
\mathscr{L}_{\beta} f(z)=\int_{0}^{\infty} d m(t) t^{\beta-1 / 2} e^{-z t}\left(\mathscr{K}_{\beta} \hat{\varphi}\right)(t),
$$

where $\mathscr{K}_{\beta}: \mathscr{L}_{2}\left(d m, \mathbb{R}_{+}\right) \rightarrow \mathscr{L}_{2}\left(d m, \mathbb{R}_{+}\right)$is the integral operator

$$
\left(\mathscr{K}_{\beta} \hat{\varphi}\right)(t)=\int_{0}^{\infty} d m(s) \oiint_{2 \beta-1}(2 \sqrt{s t}) \hat{\varphi}(s) .
$$

If we therefore define for $\beta=\beta_{1}+i \beta_{2}, \beta_{1}>\frac{1}{2}$ a linear map $j_{\beta}: \mathscr{L}_{2}\left(d m, \mathbb{R}_{+}\right) \rightarrow$ $\mathscr{H}_{\mathrm{Re} \beta}^{(2)}\left(H_{-1 / 2}\right)$ by

$$
j_{\beta}(\hat{\varphi})(z)=\frac{2^{\beta_{1}-1 / 2}}{\Gamma\left(2 \beta_{1}-1\right)^{1 / 2}} \int_{0}^{\infty} d m(t) e^{-z t} t^{\beta-1 / 2} \hat{\varphi}(t)
$$

then by Corollary $2 j_{\beta}$ is an isomorphism of the spaces $\mathscr{L}_{2}\left(d m, \mathbb{R}_{+}\right)$and $\mathscr{H}_{\beta_{1}}^{(2)}\left(H_{-1 / 2}\right)$. Relation (44) furthermore shows that

$$
\mathscr{L}_{\beta}^{\circ} j_{\beta}=j_{\beta} \circ \mathscr{K}_{\beta},
$$


and hence $\mathscr{L}_{\beta}$ and $\mathscr{K}_{\beta}$ are isomorphic. The proof of Corollary 3 is clear.

Obviously, the integral operator $\mathscr{K}_{\beta}$ in $\mathscr{L}_{2}\left(d m ; \mathbb{R}_{+}\right)$is trace class for $\operatorname{Re} \beta>\frac{1}{2}$ and its trace is given by the well known formula

$$
\operatorname{trace} \mathscr{K}_{\beta}=\int_{0}^{\infty} d m(s) \mathscr{K}_{\beta}(s, s)=\int_{0}^{\infty} d m(s) \mathfrak{F}_{2 \beta-1}(2 s) .
$$

By Theorem 2 respectively Corollary 2 the operator $\mathscr{L}_{\beta}$ in the Hilbert space $\mathscr{H}_{\beta_{1}}^{(2)}\left(H_{-1 / 2}\right)$ is trace class too with trace $\mathscr{L}_{\beta}=$ trace $\mathscr{K}_{\beta}$. In an appendix we will use the integral operator $\mathscr{K}_{\beta}$ to give a new derivation for the $\mathrm{K}-\mathrm{S}$ entropy of the Gauss map from standard perturbation theory of a symmetric operator.

\section{Trace Formulas, Fredholm Determinants and Zeta Functions}

To calculate the trace of the operator $\mathscr{K}_{\beta}$ respectively $\mathscr{L}_{\beta}$ from expression (48) we apply the formula [GR],

$$
\int_{0}^{\infty} e^{-n s} \mathfrak{F}_{2 \beta-1}(2 s) d s=2^{-(2 \beta-1)}\left(n^{2}+4\right)^{-1 / 2}\left[\left(n^{2}+4\right)^{1 / 2}-n\right]^{2 \beta-1}
$$

Since the solution $x_{n}$ of the quadratic equation $x^{2}+n x-1=0$ with $x_{n}>0$ is given by $x_{n}=-n / 2+\left(n^{2}+4\right)^{1 / 2} / 2$, and hence its inverse $x_{n}^{-1}$ by $x_{n}^{-1}=n / 2+\left(n^{2}+4\right)^{1 / 2} / 2$, which implies

$$
x_{n}+x_{n}^{-1}=\left(n^{2}+4\right)^{1 / 2},
$$

the right-hand side of (49) can be written as

$$
\text { right-hand side of }(49)=\frac{x_{n}^{2 \beta}}{1+x_{n}^{2}}
$$

Summing over $n$ then gives

$$
\operatorname{trace} \mathscr{K}_{\beta}=\sum_{n=1}^{\infty} \frac{x_{n}^{2 \beta}}{1+x_{n}^{2}} .
$$

If we compare this result with the trace of the operator $\mathscr{L}_{\beta}: A_{\infty}(D) \rightarrow A_{\infty}(D)$ in (10) we find

$$
\operatorname{trace} \mathscr{K}_{\beta}=\left.\operatorname{trace} \mathscr{L}_{\beta}\right|_{\mathscr{H}_{\mathbf{R e} \beta}^{(2)}}=\left.\operatorname{trace} \mathscr{L}_{\beta}\right|_{A \times(D)} \text {. }
$$

This is a special case of the following general result:

Theorem 4. The nuclear operators $\mathscr{L}_{\beta}: A_{\infty}(D) \rightarrow A_{\infty}(D)$ and $\mathscr{L}_{\beta}: \mathscr{H}_{\operatorname{Re} \beta}^{(2)}\left(H_{-1 / 2}\right) \rightarrow$ $\mathscr{H}_{\mathrm{Re} \beta}^{(2)}\left(H_{-1 / 2}\right)$ have the same eigenvalues, multiplicities included.

Since the proof proceeds more or less along the same chain of arguments as for the case $\beta=1$ in [MaR2] we can be rather brief. The main problem for general $\beta=\beta_{1}+i \beta_{2}, \beta_{1}>\frac{1}{2}$ is to show that every eigenfunction $f \in A_{\infty}(D)$ of $\mathscr{L}_{\beta}$ belongs to the space $\mathscr{H}_{\beta_{1}}^{(2)}\left(H_{-1 / 2}\right)$. To see this consider first the case $\beta_{1} \geqq 1$. If $\lambda f(z)=$ $\sum_{n=1}^{\infty}(1 /(z+n))^{2 \beta} f(1 /(z+n))$, we know that $f$ is holomorphic in every half plane $H_{-1+\delta}$ for $\delta>0$. For $z \in H_{-1+\delta}$ and $|z|$ large enough we certainly have $|f(1 /(z+n))|<M$ 
uniformly in $n$ and $|z|>R$, say. From the eigenvalue equation one then deduces

$$
|f(z)| \sim \frac{C}{|z|^{2 \beta_{1}-1}}
$$

for $z \rightarrow \infty$ and $z \in H_{-1+\delta}, \delta>0$, where $C=|f(0) / \lambda|$. Therefore

$$
\int_{-\infty}^{+\infty} d y|f(x+i y)|^{2}<\infty
$$

for all $x>-1+\delta, \delta>0$ and all $\beta_{1}>\frac{3}{4}$, and hence $f \in \mathscr{H}^{(2)}\left(H_{-1+\delta}\right)$ for all $\delta>0$. We have still to show that

$$
\|f\|_{\mathscr{H}_{\beta_{1}}^{2}\left(H_{-1 / 2}\right)}^{2}=\frac{1}{2 \pi} \int_{0}^{\infty} x^{2 \beta_{1}-2} d x \int_{-\infty}^{+\infty} d y\left(\left|f\left(x+i y-\frac{1}{2}\right)\right|^{2}-|f(x+i y)|^{2}\right)<\infty .
$$

From the asymptotic behaviour (53) it follows that in the half plane $H_{0}$ one has

$$
\left.|| f\left(z-\frac{1}{2}\right)\right|^{2}-|f(z)|^{2} \mid \underset{z \rightarrow \infty}{\sim} \frac{C^{\prime}}{|z|^{4 \operatorname{Re} \beta-1}} .
$$

Using polar coordinates $z=\mathrm{re}^{i \varphi}, \pi / 2 \leqq \varphi \leqq \pi / 2$ in $H_{0}$ the norm of an eigenfunction $f$ in $A_{\infty}(D)$ can be written as

$$
\|f\|_{\mathscr{F}_{\beta_{1}}^{2}\left(H_{-1 / 2}\right)}^{2}=\frac{1}{2 \pi} \int_{0}^{\infty} r^{2 \beta_{1}-1} d r \int_{-\pi / 2}^{\pi / 2} d \varphi(\cos \varphi)^{2 \beta_{1}-2}\left(\left|f\left(\mathrm{re}^{i \varphi}-\frac{1}{2}\right)\right|^{2}-\left|f\left(\mathrm{re}^{i \varphi}\right)\right|^{2}\right) .
$$

Convergence properties of the integral on the right-hand side of this expression are because of (54) the same as those of the integral

$$
\int_{R}^{\infty} r^{-\left(4 \beta_{1}-1\right)} r^{2 \beta_{1}-1} d r=\int_{R}^{\infty} r^{-2 \beta_{1}} d r
$$

which certainly exists for $\beta_{1} \geqq 1$.

This shows that any eigenfunction $f \in A_{\infty}(D)$ of the operator $\mathscr{L}_{\beta}$ belongs to the space $\mathscr{H}_{\beta_{1}}^{(2)}\left(H_{-1 / 2}\right)$ for all $\beta \in \mathbb{C}$ with $\beta_{1}=\operatorname{Re} \beta \geqq 1$. To extend this result to $\beta$ 's with $\beta_{1}>\frac{1}{2}$ we have to show that for such $\beta$ values any eigenfunction $f \in A_{\infty}(D)$ has the property that $f^{\prime}$ belongs to $\mathscr{H}_{\beta_{1}+1}^{(2)}\left(H_{-1 / 2}\right)$, since obviously $\lim _{x \rightarrow \infty} f(x+i y)=0$ and $f \in \mathscr{H}^{(2)}\left(H_{-1+\delta}\right)$ for all $\delta>0$. From the equation

$$
\lambda f^{\prime}(z)=-2 \beta \sum_{n=1}^{\infty}\left(\frac{1}{z+n}\right)^{2 \beta+1} f\left(\frac{1}{z+n}\right)-\sum_{n=1}^{\infty}\left(\frac{1}{z+n}\right)^{2 \beta+2} f^{\prime}\left(\frac{1}{z+n}\right),
$$

one deduces

$$
\left|f^{\prime}(z)\right| \underset{z \rightarrow \infty}{\sim} \frac{C}{|z|^{2 \beta_{1}}}, \quad z \in H_{-1+\delta},
$$

and hence $f^{\prime} \in \mathscr{H}^{(2)}\left(H_{-1+\delta}\right)$ for all $\delta>0$.

On the other hand we find for the $\mathscr{H}_{\beta_{1}+1}^{(2)}\left(H_{-1 / 2}\right)$-norm of $f^{\prime}$ :

$$
\left\|f^{\prime}\right\|_{\mathscr{F}_{\beta_{1}+1}^{(2)}}^{2\left(H_{-1 / 2}\right)}=\frac{1}{2 \pi} \int_{0}^{\infty} x^{2\left(\beta_{1}+1\right)-2} d x \int_{-\infty}^{+\infty} d y\left(\left|f^{\prime}\left(z-\frac{1}{2}\right)\right|^{2}-\left|f^{\prime}(z)\right|^{2}\right) .
$$


An argument analogous to the case $\beta_{1} \geqq 1$ then shows that $\left\|f^{\prime}\right\|_{\mathscr{H}_{\beta_{1}+1}^{(2)}\left(H_{-1 / 2}\right)}^{2}$ is finite if and only if $\int_{R}^{\infty} r^{2 \beta_{1}+1}\left(1 / r^{4 \beta_{1}+1}\right) d r$ exists. But $\int_{R}^{\infty} d r / r^{2 \beta_{1}}$ obviously exists for $\beta_{1}>\frac{1}{2}$. This concludes our remarks to the proof of Theorem 4 .

An immediate consequence of Theorem 4 is

Corollary 4. For any $n \in \mathbb{N}$ the following formula holds:

$$
\begin{aligned}
& \int_{0}^{\infty} d m\left(s_{n}\right) \cdots \int_{0}^{\infty} d m\left(s_{1}\right) \mathbb{F}_{2 \beta-1}\left(2 \sqrt{s_{1} s_{2}}\right) \cdots \mathfrak{F}_{2 \beta-1}\left(2 \sqrt{s_{n-1} s_{n}}\right) \mathfrak{F}_{2 \beta-1}\left(2 \sqrt{s_{n} s_{1}}\right) \\
& =\sum_{i_{1}, \ldots, i_{n}=1}^{\infty} \frac{\prod_{k=1}^{n} x_{i_{k} \cdots i_{n} i_{1} \cdots i_{k-1}}^{2 \beta}}{1-(-1)^{n} \prod_{k=1}^{n} x_{i_{k} \cdots i_{n} i_{1} \cdots i_{k-1}}^{2}}
\end{aligned}
$$

where $x_{i_{1} \ldots i_{n}}$ denotes the irrational number whose continued fraction expansion is periodic of period $n$ and whose entries are the integers $i_{1}, \ldots, i_{n}$.

Since $\mathscr{L}_{\beta}^{(1)}=-\mathscr{L}_{\beta+1}^{(0)}$ the results for $\mathscr{L}_{\beta}=\mathscr{L}_{\beta}^{(0)}$ can be applied also to the operator $\mathscr{L}_{\beta}^{(1)}$ : this operator is isomorphic to the integral operator $-\mathscr{K}_{\beta+1}$ and a formula analogous us (56) holds. A consequence of this is

\section{Corollary 5.}

$$
\begin{aligned}
& \sum_{i_{1}, \ldots, i_{n}}^{\infty} \prod_{k=1}^{n} x_{i_{k} \cdots i_{n} i_{1} \cdots i_{k-1}}^{2 \beta} \\
& \quad=\int_{0}^{\infty} d m\left(s_{n}\right) \cdots \int_{0}^{\infty} d m\left(s_{1}\right)\left[\mathfrak{F}_{2 \beta-1}\left(2 \sqrt{s_{1} s_{2}}\right) \cdots \mathfrak{F}_{2 \beta-1}\left(2 \sqrt{s_{n} s_{1}}\right)-(-1)^{n},\right. \\
& \left.\mathfrak{F}_{2 \beta+1}\left(2 \sqrt{s_{1} s_{2}}\right) \cdots \mathfrak{F}_{2 \beta+1}\left(2 \sqrt{s_{n} s_{1}}\right)\right] \\
& \quad=\operatorname{trace} \mathscr{K}_{\beta}^{n}-\operatorname{trace}\left(-\mathscr{K}_{\beta+1}\right)^{n}=\operatorname{trace} \mathscr{L}_{\beta}^{(0) n}-\operatorname{trace} \mathscr{L}_{\beta}^{(1) n} .
\end{aligned}
$$

By Corollary 5 the calculation of the infinite sum on the left-hand side has been transformed to an $n$-dimensional integral. Resulting advantages for the numerical determination of these quantities have been discussed for $\beta=1$ in [MaR1]. They apply for general $\beta$ with $\operatorname{Re} \beta>\frac{1}{2}$.

In the first chapter we had introduced a zeta function $\zeta(\beta)$ which by (13) could be expressed as the quotient of the Fredholm determinants $\operatorname{det}\left(1-\mathscr{L}_{\beta}^{(s)}\right), s=0,1$. From Grothendieck's theory of such determinants we concluded that $\zeta(\beta)$ is meromorphic in the half plane $\operatorname{Re} \beta>\frac{1}{2}$ with a simple pole at $\beta=1$. We want to extend this result now. For this we consider the operators $\mathscr{L}_{\beta}^{(s)}$ again as acting in the Banach space $A_{\infty}(D)$. It is enough to treat the case $\mathscr{L}_{\beta}^{(0)}=\mathscr{L}_{\beta}$. For this operator we can prove

Theorem 5. The map $\beta \rightarrow \mathscr{L}_{\beta}$ extends to a meromorphic function with values nuclear operators of order zero in the entire complex $\beta$ plane. Its poles, located at $\beta=(1-k) / 2$, $k=0,1, \ldots$, are all simple with residue the rank 1 operator $\mathscr{N}_{k} f(z)=\left(f^{(k)}(0)\right) / 2 k$ !.

Proof. Any $f \in A_{\infty}(D)$ has a power series expansion around the point $z=0$ which 
is uniformly convergent for $|z| \leqq \frac{1}{4}$. Hence for $|z| \leqq \frac{1}{4}$ the function

$$
f_{N}(z)=f(z)-\sum_{k=0}^{N} \frac{f^{(k)}(0)}{k !} z^{k}
$$

which obviously is in $A_{\infty}(D)$, fulfills the bound

$$
\left|f_{N}(z)\right| \leqq C|z|^{N+1} \text {. }
$$

Furthermore the map $\mathscr{P}_{N}: A_{\infty}(D) \rightarrow A_{\infty}(D)$, defined as

$$
\mathscr{P}_{N} f=f_{N},
$$

is bounded. For $\beta$ with $\operatorname{Re} \beta>\frac{1}{2}$ we can write $\mathscr{L}_{\beta} f$ also as

$$
\mathscr{L}_{\beta} f=\mathscr{L}_{\beta}\left(f-f_{N}\right)+\mathscr{L}_{\beta} f_{N} .
$$

The first term on the right-hand side of (60) can be calculated explicitly for $\operatorname{Re} \beta>\frac{1}{2}$ :

$$
\mathscr{L}_{\beta}\left(\sum_{k=0}^{N} \frac{f^{(k)}(0)}{k !} z^{k}\right)=\sum_{k=0}^{N} \frac{f^{(k)}(0)}{k !} \sum_{n=1}^{\infty}\left(\frac{1}{z+n}\right)^{2 \beta}\left(\frac{1}{z+n}\right)^{k} .
$$

But the right-hand side is simply

$$
\sum_{k=0}^{N} \frac{f^{(k)}(0)}{k !} \zeta(k+2 \beta, z+1)
$$

where $\zeta(s, z)$ denotes the zeta function of Hurwitz as defined for $\operatorname{Re} s>1$ in (39). Denote by $\mathcal{N}_{\beta}^{(k)}: A_{\infty}(D) \rightarrow A_{\infty}(D)$ the linear bounded rank 1 operator

$$
\mathcal{N}_{\beta}^{(k)} f(z)=\frac{f^{(k)}(0)}{k !} \zeta(k+2 \beta, z+1) .
$$

Then we can write the operator $\mathscr{L}_{\beta}$ for $\operatorname{Re} \beta>\frac{1}{2}$ as

$$
\mathscr{L}_{\beta}=\sum_{k=0}^{N} \mathcal{N}_{\beta}^{(k)}+\mathscr{L}_{\beta} \circ \mathscr{P}_{N}
$$

where the projector $\mathscr{P}_{N}$ of $A_{\infty}(D)$ onto the subspace $A_{\infty}^{(N)}(D)$ of functions in $A_{\infty}(D)$ vanishing in $z=0$ at least to order $N+1$ has been defined in (59).

Let us consider first the map $\beta \rightarrow \mathcal{N}_{\beta}^{(k)}$ with $\mathcal{N}_{\beta}^{(k)}$ the rank 1 operator in (61). By Hermite's representation of the Hurwitz zeta function [E]

$$
\zeta(s, z)=\frac{1}{2 z^{s}}+\frac{z^{1-s}}{s-1}+2 \int_{0}^{\infty} \frac{\sin \left[s \tan ^{-1}\left(\frac{t}{z}\right)\right]}{\left(z^{2}+t^{2}\right)^{s / 2}} \frac{d t}{e^{2 \pi t}-1},
$$

valid for $\operatorname{Re} z>0$, the function $\zeta(k+2 \beta, z+1)$ is meromorphic in the entire $\beta$-plane with only one simple pole with residue $\frac{1}{2}$ at $\beta=(1-k) / 2$ and is for fixed $\beta \neq(1-k) / 2$ a holomorphic function in $z$ for $\operatorname{Re} z>-1$. Hence the map $\beta \rightarrow \mathcal{N}_{\beta}^{(k)}$ is a rank 1 operator-valued meromorphic function in the entire complex $\beta$ plane with a simple pole at $\beta=(1-k) / 2$ and residue the rank 1 operator $\mathscr{N}^{(k)}: A_{\infty}(D) \rightarrow A_{\infty}(D)$ with

$$
\mathscr{N}^{(k)} f(z)=\frac{f^{(k)}(0)}{2 k !} .
$$


This follows from [E]

$$
\lim _{\beta \rightarrow(1-k) / 2}\left(\zeta(k+2 \beta, z+1)-\frac{1}{k+2 \beta-1}\right)=-\psi(z+1)
$$

where $\psi(z)=(d / d z) \log \Gamma(z)$ and $\Gamma$ is Eulers function. Obviously, the operators $\mathscr{N}^{(k)}$ are nilpotent for $k \geqq 1$. This shows that also the map $\beta \rightarrow \sum_{k=0}^{N} \mathcal{N}_{\beta}^{(k)}$ can be meromorphically extended to the entire complex $\beta$-plane with simple poles at the points $\beta=(1-k) / 2,0 \leqq k \leqq N$ and corresponding residues $\mathscr{N}^{(k)}$.

Let us next discuss the operator $\mathscr{L}_{\beta} \circ \mathscr{P}_{N}$. Since by (58) for any $f \in A_{\infty}(D)$ the function $\left(\mathscr{P}_{N} f\right)(z)=f_{N}(z)$ fulfills $\left|\mathscr{P}_{N} f(z)\right| \leqq C \cdot|z|^{N+1}$ for all $|z| \leqq \frac{1}{4}$ we see that in the representation

$$
\mathscr{L}_{\beta} \circ \mathscr{P}_{N} f=\sum_{n=1}^{\infty}\left(\frac{1}{z+n}\right)^{2 \beta} f_{N}\left(\frac{1}{z+n}\right)
$$

the sum converges uniformly and absolutely in $D$ for all $\beta \in \mathbb{C}$ with $2 \operatorname{Re} \beta+N+1>1$, that means $\operatorname{Re} \beta>-(N / 2)$. This shows, that the operator $\mathscr{L}_{\beta}: A_{\infty}^{(N)}(D) \rightarrow A_{\infty}(D)$ is nuclear of order zero for all $\beta$ with $\operatorname{Re} \beta>-(N / 2)$. Since $\mathscr{P}_{N}: A_{\infty}(D) \rightarrow A_{\infty}^{(N)}(D)$ is bounded the operator $\mathscr{L}_{\beta} \circ \mathscr{P}_{N}$ is also nuclear of order zero for $\operatorname{Re} \beta>-(N / 2)$. Obviously, also the finite-rank operator $\sum_{k=0}^{N} \mathcal{N}_{\beta}^{(k)}$ is nuclear of order zero away from the points $\beta=(1-k) / 2, k=0, \ldots, N$. All this being true for general $N$ the proof of Theorem 5 is finished.

Interesting by itself as we see later is the following

Corollary 6. The function $g(\beta)=$ trace $\mathscr{L}_{\beta}$ extends to a meromorphic function in the entire $\beta$ plane with only one simple pole at the point $\beta=\frac{1}{2}$ with residue $\frac{1}{2}$.

Proof. By (62) we have for $\operatorname{Re} \beta>-(N / 2), \beta \neq(1-k) / 2$

$$
\operatorname{trace} \mathscr{L}_{\beta}=\sum_{k=0}^{N} \operatorname{trace} \mathscr{N}_{\beta}^{(k)}+\operatorname{trace} \mathscr{L}_{\beta} \circ \mathscr{P}_{N} \text {. }
$$

But the trace of the rank 1 operator $\mathcal{N}_{\beta}^{(k)}$ is given by

$$
\operatorname{trace} \mathcal{N}_{\beta}^{(k)}=\left.\frac{1}{k !} \frac{d^{k}}{d z^{k}} \zeta(2 \beta+k, z+1)\right|_{z=0}
$$

Using next the formulas

$$
\frac{d}{d z} \zeta(s, z)=-s \zeta(s+1, z)
$$

respectively

$$
\zeta(s, 1)=\zeta_{R}(s)=\sum_{n=1}^{\infty} \frac{1}{n^{s}}
$$

we find for $k \geqq 1$,

$$
\operatorname{trace} \mathscr{N}_{\beta}^{(k)}=\frac{(-1)^{k}}{k !}(2 \beta+k) \cdots(2 \beta+2 k-1) \zeta_{R}(2 \beta+2 k)
$$


respectively

$$
\operatorname{trace} \mathcal{N}_{\beta}^{(0)}=\zeta_{R}(2 \beta) \text {. }
$$

Since Riemann's zeta function $\zeta_{R}(s)$ in (69) can be extended to the entire $s$-plane with a single simple pole with residue 1 at $s=1$ we conclude that trace $\mathscr{N}_{\beta}^{(k)}$ for $k \geqq 1$ is holomorphic in the entire $\beta$-plane and trace $\mathcal{N}_{\beta}^{(0)}$ is meromorphic in $\beta$ with a simple pole at $\beta=\frac{1}{2}$ with residue $\frac{1}{2}$. Since on the other hand $\mathscr{L}_{\beta} \circ \mathscr{P}_{N}$ is nuclear of order zero and holomorphic in $\beta$ for $\operatorname{Re} \beta>-(N / 2)$ we find that the function $g(\beta)$ is meormorphic in this region with a simple pole at $\beta=\frac{1}{2}$ and residue $\frac{1}{2}$. Since $N$ was completely arbitrary the proof of Corollary 6 is finished. Another consequence of Theorem 5 is

Corollary 7. The Fredholm determinant $\operatorname{det}\left(1-\mathscr{L}_{\beta}\right)$ extends to a meromorphic function in the $\beta$-plane whose only poles are at the points $\beta_{k}=(1-k) / 2, k=0,1,2, \ldots$ which furthermore are simple.

This follows from the formula [G]

$$
\operatorname{det}\left(1-\mathscr{L}_{\beta}\right)=\sum_{r=0}^{\infty}(-1)^{r} \operatorname{trace} \Lambda_{r} \mathscr{L}_{\beta},
$$

where $\Lambda_{r} \mathscr{L}_{\beta}$ denotes the $r$-fold exterior product of the linear operator $\mathscr{L}_{\beta}$ in the space $A_{\infty}(D)$ together with the representation $\mathscr{L}_{\beta}=\sum_{k=0}^{N} \mathcal{N}_{\beta}^{(k)}+\mathscr{L}_{\beta} \circ \mathscr{P}_{N}$ in (62), where $\mathcal{N}_{\beta}^{(k)}$ is an operator of rank 1 which as a function of $\beta$ behaves for $\beta \rightarrow \beta_{k}=$ $(1-k) / 2$ as $\mathscr{N}^{(k)} /\left(\beta-\beta_{k}\right)$ with $\mathscr{N}^{(k)}$ defined in (64).

Remark. In the "basis" of $A_{\infty}(D)$ spanned by the functions $f_{k}$ with $f_{k}(z)=$ $\zeta(2 \beta+k, z+1), k=0,1,2, \ldots$ the operator $\mathscr{L}_{\beta}$ can be represented for $\operatorname{Re} \beta>\frac{1}{2}$ by a matrix $\mathbb{M}=\mathbb{M}_{k, \ell}(\beta), k, \ell=0,1,2, \ldots$ with

$$
\mathbf{M}_{k, \ell}(\beta)=\frac{(-1)^{k}}{k !} \frac{\Gamma(2 \beta+\ell+k)}{\Gamma(2 \beta+\ell)} \zeta_{R}(2 \beta+l+k) .
$$

From this one concludes that all matrix elements $\mathbb{M}_{k, \ell}(\beta)$ with $k \geqq 1$ can be analytically continued to the entire $\beta$-plane whereas the elements $\mathbb{M}_{0, \ell}(\beta)=\zeta_{R}(2 \beta+\ell)$ are meromorphic in the $\beta$-plane with simple poles at the points $\beta=\beta_{\ell}=(1-l) / 2$. This gives a formal proof of our results for the operator $\mathscr{L}_{\beta}$.

Corollary 7 now implies

Theorem 6. The function $\zeta(\beta)=\exp \sum_{n=1}^{\infty} \frac{Z_{n}(\beta)}{n}$ with $Z_{n}(\beta)=\sum_{x \in \operatorname{Fix}} \prod_{T^{n}}^{n-1}\left(T_{k=0}^{k} x\right)^{2 \beta}$ extends for the Gauss map as a meromorphic function to the entire $\beta$ plane. $\zeta$ has trivial zeros at $\beta=0$ and $\beta=\frac{1}{2}$. Its nontrivial zeros are at $\beta=\beta_{\ell}$ such that $-\mathscr{L}_{\beta_{+}+1}$ has eigenvalue $\lambda=1$, its nontrivial poles are at $\beta=\beta_{k}$ such that $\mathscr{L}_{\beta_{k}}$ has $\lambda=1$ as an eigenvalue. There are no poles besides $\beta=1$ on the real axis $\beta \geqq 1$ and no zeros for $\beta \geqq 0$.

Proof. Apply Corollary 7 to formula (13).

Remark. We expect besides $\beta=1$ no pole on the entire real axis. 
Another trivial application of the above results concerns analyticity properties of the following function $\zeta_{M}$ :

$$
\zeta_{M}(\beta)=\sum_{n=1}^{\infty} x_{n}^{\beta}
$$

where $x_{n}$ denotes the irrational number which has periodic continued fraction expansion of period 1 with entry $n$ as defined after relation (49). It is clear that asymptotically for large $n x_{n}$ behaves like $n^{-1}$, and hence $\zeta_{M}(\beta)$ should be somehow related to Riemann's zeta function $\zeta_{R}(\beta)=\sum_{n=0} n^{-\beta}$. Corollary 6 implies

Proposition 2. The function $\zeta_{M}(\beta)$ extends as a meromorphic function to the entire $\beta$-plane with the only poles at the points $\beta= \pm 1$ with residue 1 .

Proof. Since by Corollary 1 ,

$$
\zeta_{M}(2 \beta)=Z_{1}(\beta)=\operatorname{trace} \mathscr{L}_{\beta}^{(0)}-\operatorname{trace} \mathscr{L}_{\beta}^{(1)}=\operatorname{trace} \mathscr{L}_{\beta}+\operatorname{trace} \mathscr{L}_{\beta+1},
$$

we get from Corollary 6 that $\zeta_{M}(2 \beta)$ extends to a meromorphic function in the entire complex $\beta$-plane with simple poles at the points $\beta= \pm \frac{1}{2}$ and residue $\frac{1}{2}$. From this Proposition 2 follows.

We have seen that formally the operator $\mathscr{L}_{\beta}$ can be represented by the matrix $\mathbb{M}$ with

$$
\mathbb{M}_{k, \ell}(\beta)=\frac{(-1)^{k}}{k !} \frac{\Gamma(2 \beta+\ell+k)}{\Gamma(2 \beta+\ell)} \zeta_{R}(2 \beta+\ell+k) .
$$

By using this matrix the trace of $\mathscr{L}_{\beta}$ can be expressed as

$$
\operatorname{trace} \mathscr{L}_{\beta}=\sum_{k=0}^{\infty} \frac{(-1)^{k}}{k !} \frac{\Gamma(2 \beta+2 k)}{\Gamma(2 \beta+k)} \zeta_{R}(2 \beta+2 k) \text {. }
$$

This series is not absolutely convergent so that one has to be rather careful to calculate its values.

Since the function $\zeta_{M}(\beta)$ in (74) can be expressed as

$$
\zeta_{M}(2 \beta)=\operatorname{trace} \mathscr{L}_{\beta}+\operatorname{trace} \mathscr{L}_{\beta+1},
$$

we find after a simple calculation using representation (75)

$$
\zeta_{M}(\beta)=\beta \sum_{k=0}^{\infty} \frac{(-1)^{k}}{k !} \frac{\Gamma(\beta+2 k)}{\Gamma(\beta+k+1)} \zeta_{R}(\beta+2 k) .
$$

This representation can be used indeed to rederive the analyticity properties of the function $\zeta_{M}$. We are going to show namely that

$$
\zeta_{M}(\beta)=\zeta_{R}(\beta)-\beta \zeta_{R}(\beta+2)+\Psi(\beta),
$$

where $\Psi(\beta)$ is holomorphic in the entire $\beta$-plane. Corollary 5 shows that for $n=1$ and $\operatorname{Re} \beta>1$,

$$
\zeta_{M}(\beta)=\int_{0}^{\infty} \frac{d s}{e^{s}-1}\left[\mathfrak{F}_{\beta-1}(2 s)+\mathfrak{F}_{\beta+1}(2 s)\right] .
$$


Using the functional equation for Bessel functions [GR],

$$
\mathfrak{F}_{v+1}(z)+\mathfrak{F}_{v-1}(z)=\frac{2 v}{z} \mathfrak{F}_{v}(z),
$$

this simplifies to

$$
\zeta_{M}(\beta)=\beta \int_{0}^{\infty} \frac{d s}{e^{s}-1} \frac{\mathfrak{F}_{\beta}(2 s)}{s} .
$$

If we introduce the finite power series $\mathfrak{\mho}_{\beta}^{(N)}(2 s)$,

$$
\mathfrak{F}_{\beta}^{(N)}(2 s)=s^{\beta} \sum_{k=0}^{N} \frac{(-1)^{k} s^{2 k}}{k ! \Gamma(k+\beta+1)}
$$

we get for $\operatorname{Re} \beta>1$ the following representation for $\zeta_{M}$ :

$$
\zeta_{M}(\beta)=\beta \int_{0}^{\infty} \frac{d s}{e^{s}-1} \frac{\mathfrak{F}_{\beta}^{(N)}(2 s)}{s}+\beta \int_{0}^{\infty} \frac{d s}{e^{s}-1} \frac{\mathfrak{F}_{\beta}(2 s)-\mathfrak{F}_{\beta}^{(N)}(2 s)}{s} .
$$

The first integral in (81) can be performed explicitly to give

$$
\begin{aligned}
\beta \int_{0}^{\infty} \frac{d s}{e^{s}-1} \frac{\mathfrak{F}_{\beta}^{(N)}(2 s)}{s} & =\beta \sum_{k=0}^{N} \frac{(-1)^{k}}{k !} \frac{\Gamma(\beta+2 k)}{\Gamma(\beta+k+1)} \zeta_{R}(\beta+2 k) \\
& =\zeta_{R}(\beta)-\beta \zeta_{R}(\beta+2)+\Psi^{(N)}(\beta) .
\end{aligned}
$$

The function $\Psi^{(N)}(\beta)$ is obviously holomorphic in the entire $\beta$-plane for all $N \geqq 2$. The second integral in (81) defines a function $\Phi^{(N)}(\beta)$ which is holomorphic in $\beta$ for all $\beta$ with $\operatorname{Re} \beta>-(2 N+1)$ since

$$
\mathfrak{F}_{\beta}(2 s)-\mathscr{F}_{\beta}^{(N)}(2 s) \underset{s \rightarrow 0}{\sim} s^{\beta+2(N+1)} .
$$

This shows that the function $\zeta_{M}$ can indeed be represented as

$$
\zeta_{M}(\beta)=\zeta_{R}(\beta)-\beta \zeta_{R}(\beta+2)+\Psi(\beta)
$$

where

$$
\Psi(\beta)=\Psi^{(N)}(\beta)+\Phi^{(N)}(\beta)
$$

is independent of $N$ for $N \geqq 2$ and hence holomorphic in the entire $\beta$-plane.

Since the function $\zeta_{M}$ is defined by the numbers $[n]$ which have nice arithmetic properties like periodic continued fractions, one could wonder if $\zeta_{M}$ does not fulfill some sort of functional equation. Unfortunately we cannot say much to this problem at present.

Let us add some remarks concerning the transfer operator $\mathscr{L}_{\beta}$ and its Fredholm determinant $\operatorname{det}\left(1-\mathscr{L}_{\beta}\right)$. Quite recently $\mathrm{M}$. Pollicott gave a new approach to Selberg's theory of compact surfaces of constant negative curvature through the transfer operator method [P]. He applied this method to the Bowen-Series maps, analytic expanding Markov maps of the boundary of the unit disk, which have been used by these authors to construct symbolic dynamics for the geodesic flow on these surfaces [BoS]. It turns out that there exists a close connection between 
the Fredholm determinant of the transfer operator for these boundary maps and the Selberg zeta function for the flow on the surface. Its poles are known to determine the spectrum of the Laplacian on that surface and this means that also the transfer operator for the corresponding Bowen-Series map determines this spectrum. The operator however is completely determined by the classical geodesic flow on the surface.

From this one should expect that very similar things are true for the modular surface: the Bowen-Series map in this case is just the Gauss map $T$ in (1) whose transfer operator we have studied in this paper. One could therefore hope that there is also a close relation of the function $\zeta(\beta)$ in Theorem 6 and the Selberg zeta function for the geodesic flow on the modular surface. Indeed, the pole $\beta=1$ of $\zeta(\beta)$ corresponds, when translating Pollicott's formulas to the present case, just to the lowest lying eigenvalue $\lambda=0$ of $-\Delta$ for the modular surface with $f=$ const. as eigenfunction.

Let us finally come back to our discussion of phase transitions in hyperbolic dynamical systems in Sect. 1. There we argued that hyperbolic dynamical systems with a finite Markov partition cannot have such a phase transition, that is a singularity in the function $P(\beta)$ for real $\beta$. For the Gauss map, whose minimal Markov partition is infinite, this is not true. From what we have found for the transfer operator $\mathscr{L}_{\beta}$ it follows that $P(\beta)$ has a logarithmic singularity at $\beta=\frac{1}{2}$, and henceforth $T$ has a phase transition for "finite" temperature. This follows from the behaviour of $\mathscr{L}_{\beta}$ for $\beta \rightarrow \beta_{0}=\frac{1}{2}$ determined by relations (64) and (65):

$$
\lim _{\beta \rightarrow \beta_{0}}\left(\beta-\beta_{0}\right) \mathscr{L}_{\beta}=\mathscr{N}^{(0)} \text {. }
$$

This shows that for real $\beta$ the leading eigenvalue $\lambda_{1}(\beta)$ behaves for $\beta \rightarrow \beta_{0}$ like

$$
\lambda_{1}(\beta) \sim \frac{1}{\beta-\beta_{0}} \cdot \frac{1}{2}+\cdots,
$$

where $\frac{1}{2}$ is the eigenvalue of the operator $\mathscr{N}^{(0)}$. All the other eigenvalues $\lambda_{i}(\beta)$ have a finite value for $\beta=\beta_{0}=\frac{1}{2}$. This is just what one expected from Corollary 6 .

\section{Appendix. The K-S Entropy of $T$ Through the Thermodynamic Formalism}

From the variational principle (9) together with Pesin's identity

$$
h_{\mathrm{K}-\mathrm{s}}(T)=\int_{0}^{1} d \mu_{G}(x) \log \left|T^{\prime}(x)\right|,
$$

where $d \mu_{G}=(1 / \log 2) \frac{1}{(x+1)} d x$ denotes normalized Gauss measure for $T$, one derives the formula [Ra], [RaB]:

$$
h_{\mathrm{K}-\mathrm{S}}(T)=-\left.\frac{d}{d \beta} P(\beta)\right|_{\beta=1} .
$$

Since $P(\beta)=\log \lambda_{1}(\beta)$ with $\lambda_{1}(\beta)$ the leading eigenvalue of the transfer operator $\mathscr{L}_{\beta}$ in Proposition 1 we find

$$
h_{\mathrm{K}-\mathrm{S}}(T)=-\left.\lambda_{1}^{\prime}(\beta)\right|_{\beta=1}
$$


because $\lambda_{1}(1)=1$. Since for real $\beta>\frac{1}{2}$ the eigenvalue $\lambda_{1}(\beta)$ is simple it is analytic in $\beta$ in a neighbourhood of $\beta=1$, and hence

$$
\lambda_{1}(\beta)=1+(\beta-1) \lambda_{1}^{\prime}(1)+\cdots
$$

is a convergent power series for $|\beta-1|$ small enough. Since $\sigma\left(\mathscr{L}_{\beta}\right)=\sigma\left(\mathscr{K}_{\beta}\right)$ with $\mathscr{K}_{\beta}$ the integral operator in Theorem 3 , standard perturbation theory applied to the selfadjoint operator $\mathscr{K}_{\beta}, \beta$ real, gives

$$
\lambda_{1}^{\prime}(1)=\left(\varphi_{1}, \mathscr{K}^{\prime}{ }_{1} \varphi_{1}\right)
$$

where $\mathscr{K}_{1}^{\prime}$ is defined through the power series expansion of the operator $\mathscr{K}_{\beta}$ as

$$
\mathscr{K}_{\beta}=\mathscr{K}_{1}+(\beta-1) \mathscr{K}_{1}^{\prime}+\cdots,
$$

$\varphi_{1}$ is the normalized eigenfunction of $\mathscr{K}_{1}$ with eigenvalue $\lambda_{1}(1)$ and (,) denotes the usual scalar product in the Hilbert space $\mathscr{L}_{2}\left(d m, \mathbb{R}_{+}\right)$. The eigenfunction $\varphi_{1}$ is known explicitly [MaR1]:

$$
\varphi_{1}(s)=\frac{1}{\sqrt{\log 2}} s^{-1 / 2}\left(1-e^{-s}\right) .
$$

The operator $\mathscr{K}_{1}^{\prime}$ on the other hand is defined through the kernel

$$
K_{1}^{\prime}(s, t)=\left.2 \frac{d}{d v} \mathfrak{F}_{v}(2 \sqrt{s t})\right|_{v=1}
$$

This kernel can be written as [AS]

$$
K_{1}^{\prime}(s, t)=2\left[\mathfrak{F}_{1}(2 \sqrt{s t}) \log \sqrt{s t}-\sum_{m=0}^{\infty}(-1)^{m} \sqrt{s t^{2 m+1}} \frac{\psi^{(m+2)}}{m !(m+1) !}\right] .
$$

Using this representation one finds

$$
\mathscr{K}^{\prime}{ }_{1} \varphi_{1}(s)=\frac{1}{\sqrt{\log 2}} \sum_{k=0}^{\infty} \frac{(-1)^{k}}{(k+1) !} s^{k+1 / 2}(\log s+\psi(k+1)-2 \psi(k+2)) .
$$

Inserting this into formula (A4) leads to

$$
\lambda_{1}^{\prime}(1)=\frac{2}{\log 2} \sum_{k=0}^{\infty} \frac{(-1)^{k+1}}{k+1}(\psi(k+2)-\psi(k+1)) .
$$

Since $\psi(k+2)=\psi(k+1)+1 /(k+1)$ we get

$$
\lambda_{1}^{\prime}(1)=\frac{2}{\log 2} \sum_{k=1}^{\infty} \frac{(-1)^{k}}{k^{2}}=-\frac{\pi^{2}}{6 \log 2},
$$

and therefore finally

$$
h_{\mathrm{K}-\mathrm{S}}(T)=\frac{\pi^{2}}{6 \log 2} .
$$

Obviously, this result can be derived directly from Pesin's identity (A1). The approach presented above however is more general in that it allows to determine also the higher derivatives of $P(\beta)$ at $\beta=1$ which themselves are again interesting invariants of the system $T: I \rightarrow I$. 
Acknowledgements. Work on this paper has been done during a stay at the KFA Jülich and at the MPI für Mathematik at Bonn. Financial support by both of these institutions is cordially acknowledged.

\section{References}

[AS] Abramowitz, M., Stegun, I.: Handbook of Math. Functions, p. 362. New York: Dover 1972

[Bo] Bowen, R.: Equilibrium states and the ergodic theory of Anosov diffeomorphisms, Lecture Notes in Mathematics, vol. 470. Berlin, Heidelberg, New York: Springer 1975

[BoR] Bowen, R., Ruelle, D.: The ergodic theory of Axiom A flows. Invent. Math. 29, 181-202 (1975)

[BoS] Bowen, R., Series, C.: Markov maps associated to Fuchsian groups. Publ. I.H.E.S. 50, 153-170 (1979)

[D] Duren, P.: Theory of $H^{p}$-spaces. New York: Academic Press 1970

[E] Erdelyi, A. et al.: Higher Transcendental Functions, vol 1, p. 26. New York: McGraw Hill 1953

[G] Grothendieck, A.: La théorie de Fredholm. Bull. Soc. Math. France 84, 319-384 (1956)

[GR] Gradshteyn, I., Ryzhik, I.: Table of integrals, series and products. New York: Academic Press 1965

[K] Keane, M.: Strongly mixing g-measures. Invent. Math. 16, 309-324 (1972)

[LaM] Lasota, A., Mackey, M.: Probabilistic properties of deterministic systems. Cambridge: Cambridge University Press 1985

[Ma1] Mayer. D.: A zeta function related to the continued fraction transformation. Bull. Soc. Math. France 104, 195-203 (1976)

[Ma2] Mayer, D.: The Ruelle-Araki transfer operator in classical statistical mechanics, Lecture Notes in Physics, vol 123. Berlin, Heidelberg, New York: Springer 1980

[Ma3] Mayer, D.: On the location of poles of Ruelle's zeta function. Lett. Math. Phys. 14, 105-115 (1987)

[Ma4] Mayer, D.: Continued fractions and related transformations. Lectures at the Topical Meeting on Hyperbolic Geometry and Ergodic Theory, ICTP-Trieste, Italy (1989)

[Ma5] Mayer, D.: Approach to equilibrium for locally expanding maps in $\mathbb{R}^{k}$. Commun. Math. Phys. 95, 1-15 (1984)

[MaR1] Mayer, D., Roepstorff, G.: On the relaxation time of Gauss' continued fraction map I. The Hilbert space approach. J. Stat. Phys. 47, 149-171 (1987)

[MaR2] Mayer, D., Roepstorff, G.: On the relaxation time of Gauss' continued fraction map II. The Banach space approach. J. Stat. Phys. 50, 331-344 (1988)

[Pa] Parry, W.: The $\beta$-function is a complete inariant for finite equivalence. Warwick University. Preprint, June 1984

[PaT] Parry, W., Tuncel, S.: On the classification of Markov chains by finite equivalence. Ergod. Th. Dyn. Syst. 1, 303-335 (1981)

[P] Pollicott, M.: The spectral analysis of the Riemann surface through Perron-Frobenius type operators. Unpubl. Notes

[Ra] Rand, D.: The singularity spectrum for hyperbolic Cantor sets and attractors. Arizona University. Preprint (1986)

[RaB] Rand, D., Bohr, T.: The entropy function for characteristic exponents. Physica D 25, 387-398 (1987)

[Ru1] Ruelle, D.: Thermodynamic Formalism, Encycl. of Math. and its Appl. vol 5. Reading Mass.: Addison-Wesley 1978

[Ru2] Ruelle, D.: A measure associated with Axiom A attractors. Am. J. Math. 98, 619-654 (1976)

[W1] Walters, P.: Ruelle's operator theorem and $g$-measures. Trans. Am. Math. Soc. 214, 375-387 (1975)

[W2] Walters, P.: A variational principle for the pressure of continuous transformations. Am. J. Math. 97, 937-971 (1975)

Communicated by J.-P. Eckmann 
